\title{
PROLIFERATION AND PHAGOCYTOSIS.
}

By F. B. MALLORY, M. D.

(From the Sears Pathological Laboratory, Harvard University Medical Schood.)

The effects which injurious agents, especially the toxines secreted by bacteria, produce on tissues are manifested in four different ways:

1 , by degeneration or necrosis of cells;

2 , by exudation from the blood-vessels;

3 , by proliferation of cells; and

4 , by phagocytosis, this term being used here to mean the inclusion and digestion of certain cells by other cells.

These four processes may occur separately, or in various combinations and proportions. The first two processes are generally accepted and taught. It is with reference to the last two, proliferation and phagocytosis, that I wish to present a certain amount of evidence bearing on the character of the cells concerned, on the nature of the toxines which excite the processes, on the duration and termination of the lesions, and on the relation of the processes to repair.

The view that toxines can cause various cells to proliferate, and certain cells to become phagocytic for other cells, was first forcibly called to my attention by a histological study of the lesions of typhoid fever published in this Journat in $1898 .^{1} \quad$ Since then I have studied these processes in various diseases in nearly a thousand autopsies occurring at the Boston City Hospital during the past three years.

What I wish to show is that strong toxines cause degeneration or necrosis of cells, and exudation, while dilute and weak toxines produce proliferation and phagocytosis. In support of this hypothesis I will cite in part the result of the study of large numbers of cases of certain diseases, in part a limited number of typical and of unusual lesions.

1 Journal of Experimental Medicine, 1898, iii, p. 611. 
Certain organisms, such as Staphylococcus pyogenes aureus and Bacillus diphtheriæ, produce strong toxines which act quickly, and chiefly locally: the effect on the tissues is to produce necrosis and exudation. Other organisms, such as Bacillus typhosus, produce mild toxines which act slowly, and cause proliferation or phagocytosis or both.

The stronger toxines, however, can produce these same processes of proliferation and phagocytosis when sufficiently dilute. The best example of this which I have found was in the kidneys of a girl five years old who died in four days with an acute myositis of the right thigh, due to Staphylococcus pyogenes aureus. The autopsy was made one hour after death. The femur was not affected. There were multiple miliary abscesses in the lungs, heart, and kidneys. . In the kidneys they occurred for the most part in the pyramids, especially towards the bases. Very few of the foci were softened: most of them consisted of areas of necrosis with more or less infiltration with polynuclear leukocytes. Judged by inoculation experiments in rabbits, the lesions were not over 48 hours old.

Many of the lymphatics adjoining these beginning abscesses and running towards the bases of the pyramids were dilated and filled with numerous large phagocytic cells, together with a few lymphoid and plasma cells, and polynuclear leukocytes. The inclusions in the phagocytic cells consisted principally of polynuclear leukocytes and lymphoid cells. Careful study of these lymphatics showed occasional mitosis of the lining endothelium, and much more frequently migration of the endothelial cells through the walls into the adjoining connective tissue. The endothelial cells pass through the wall of a lymphatic in exactly the same way that the polynuclear leukocytes do; namely, a process of the nucleus surrounded by a minimum of protoplasm pierces the wall first, and the rest of the nucleus and the protoplasm gradually follow. After the endothelial cells contained inclusions they still showed the changes of form characteristic of amœboid motion, but none was found in the act of emigration.

In diphtheria the chief lesions are focal and occur for the most part in the air passages, in the immediate vicinity of the bacilli of diph- 
theria; but there are other lesions due to the diffusion of a small amount of the toxine through the blood and lymph circulations. The most interesting of the latter are found in the lymph nodules of the tonsils, spleen, and gastro-intestinal tract. They consist of a moderate proliferation of the cells lining the reticulum. This proliferation usually occurs in the centres of the lymph nodules, but sometimes is diffuse or eccentric. Even more marked than the proliferation is the incorporation of the lymphoid cells of the lymph nodules by the cells lining the reticulum and by the new cells formed from them. Phagocytosis like proliferation usually begins in the centres of the lymph nodules and spreads peripherally, but may occur diffusely. In advanced lesions all or nearly all of the lymphoid cells in a lymph nodule may be destroyed. In such cases the centre of a lymph nodule shows a clump of epithelioid cells with the protoplasm more or less fused and containing no inclusions because all of the incorporated cells have been digested, and others are not within reach. Towards the periphery phagocytic cells containing partially digested cells are found, while at the periphery the phagocytic cells are stuffed with lymphoid cells still in a fair state of preservation. The large cells in the centres of the lymph nodules sometimes undergo necrosis, probably in consequence of lack of nutrition.

This brief statement of the proliferation and phagocytosis occurring in diphtheria is based on the histological study of 220 cases of diphtheria, of which a full report will be published shortly by Councilman, Pearce, and myself.

Pratt $^{2}$ has recently shown in a study of 50 cases of acute lobar pneumonia that Micrococcus lanceolatus causes marked proliferation of the cells lining the alveoli, the pleural cavities, and the lymphatics: these newly-formed cells are very phagocytic, incorporating and digesting polynuclear leukocytes, lymphoid and plasma cells, and red bloodglobules. The proliferation is more marked in the early and in the late stages of the disease than in the middle stage when the exudation of polynuclear leukocytes is abundant.

${ }^{2}$ Contributions to the Science of Medicine dedicated by his Pupils to William Henry Welch on the Twenty-fifth Anniversary of his Doctorate, p. 265. Baltimore, 1900. 
The acute lesions of the glomeruli of the kidney throw much light on the question of proliferation due to bacterial toxines. The organisms most frequently concerned are Micrococcus lanceolatus and Streptococcus pyogenes. The primary lesion from which the toxine escapes into the circulation is often an acute endocarditis; or the toxine may be secreted directly in the circulating blood in consequence of a septicæmia only. In other cases the primary lesion and the source of the toxine are more remote from the circulation. The lesions produced in the glomeruli vary from necrosis and exudation to pure proliferation.

The toxine secreted by Micrococcus lanceolatus causes proliferation almost exclusively of the endothelial cells lining the capillaries of the glomeruli. In one case (No. 1178) of acute endocarditis, in which death occurred at the end of four weeks, the kidneys showed an essentially pure type of acute proliferative intra-capillary glomerulonephritis. Mitotic figures in the endothelial cells were numerous; repeatedly two were found in one section of a single glomerulus.

With Streptococcus pyogenes the lesion produced in the glomeruli varies greatly; sometimes it is entirely exudative; at other times there is marked proliferation of the capsular epithelium or of the capillary endothelium: proliferation and exudation are often combined in various proportions.

In one case (B. C. H., No. 98.67) of streptococcus endocarditis and septicæmia there was marked proliferation of the capsular epithelium and also in places of the epithelium at the beginning of the tubules; in one place two mitotic figures in the renal epithelium were found side by side. Many mitoses were found in the capsular epithelium. In some of the capsular spaces there were a little fibrin and a few polynuclear leukocytes. Occasionally phagocytic cells were found within the capsular space: the inclusions consisted of polynuclear leukocytes and red blood-globules.

Certain organisms, such as the typhoid and tubercle bacilli, produce mild toxines which act slowly and usually produce proliferation only, but under certain conditions, especially when the organisms are massed together in large numbers, the toxines may be concentrated and produce necrosis and a purulent exudation. 
In typhoid fever a mild diffusible toxine is formed which causes great proliferation of the endothelial cells lining the lymphatics, and the reticulum of the lymphoid tissue of the intestine and the mesenteric lymph nodes; it also causes proliferation of the endothelial cells lining the blood-vessels of the intestine, liver, and spleen. All of these newly formed cells are extremely phagocytic and incorporate lymphoid and plasma cells, polynuclear lenkocytes, and red blood-corpuscles. Phagocytic cells formed within the blood-vessels are often carried by the circulation to the liver when they give rise to miliary infarctions by blocking up the capillaries. In the spleen the phagocytic cells may occlude the blood sinuses, giving rise to necroses.

Occasionally, and almost invariably following an attack of typhoid fever, the typhoid bacillus finds suitable conditions for abundant growth. Under these circumstances it produces a marked local reaction, namely, necrosis and purulent exudation like the more virulent organisms already mentioned.

The tubercle bacillus produces a variety of lesions. The typical lesion is of course the miliary tubercle, a clump of epithelioid cells produced by proliferation from endothelial and connective tissue cells. In some situations, as in the lungs, epithelial cells may also take part in the proliferation. Giant cells may or may not be formed. Ordinarily these epithelioid cells show little evidence of phagocytosis, but in certain situations, such as in the meninges of the brain, and in the lymph sinuses of lymph nodes, there may occur a very extensive diffuse proliferation of the endothelial cells which distend the sinuses widely and show marked phagocytic properties; so that an early diffuse tuberculosis of a bronchial lymph node may very closely resemble a mesenteric lymph node of the early, hyperplastic stage of typhoid fever.

On the other hand, in caseous pneumonia, the exudation is often as marked as the proliferation; and in certain rare cases the tubercle bacillus acts like the pus organisms and produces a purulent exudation. In these cases the organisms are present in great numbers, and the toxine is probably much more concentrated than usual.

In the lesions thus far considered, proliferation of endothelial cells 
has played a very important part. It can be caused apparently by a great variety of toxines; several examples have been given. This proliferation always takes place by mitosis; careful search of fresh, well preserved tissue has always shown enough typical mitotic figures to account for the newly formed cells. In all of the situations in which the endothelium proliferates, except the glomeruli, the newly formed cells are extremely phagocytic. They incorporate a certain definite group of cells, namely, polynuclear leukocytes, lvmphoid and plasma cells, and red blood-globules; they never incorporate epithelial or other endothelial cells. The included cells seem normal at the time they are taken up; occasionally the lymphoid cells have been in mitosis. After inclusion for a time in the phagocytic cells they show more or less evidence of degeneration; the nucleus first stains intensely, then dissolves more or less irregularly, and finally disappears.

The kind of cells which a phagocyte incorporates, depends on its situation in the tissue; if it lies in lymphoid tissue, it takes up lymphoid cells; if in a blood sinus of the spleen, it encloses red bloodglobules, often a dozen or more.

In acute proliferative intracapillary glomerulonephritis I have never been able to find any evidence of phagocytosis.

Phagocytic cells necessarily have the power of amoboid motion, otherwise they could not incorporate other cells. In one very perfectly preserved case, the first mentioned in this paper, the young endothelial cells formed by proliferation from the lining endothelium of the lymphatics were found actively emigrating from the vessels into the surrounding tissue.

Under certain circumstances bacterial toxines cause proliferation also of epithelial cells. This is shown most convincingly in cases of acute capsular glomerulonephritis; mitotic figures are easily demonstrable and are often comparatively numerous. Occasionally the more highly differentiated epithelial cells at the beginning of the renal tubules also show active proliferation. Rarely these new-formed cells in the capsular spaces contain inclusions, usually polynuclear leukocytes or red blood-globules. They probably would contain more inclusions if the proper cells were within reach. Proliferation of 
epithelium also takes place in pneumonia in the alveoli and in the pleural cavities; mitotic figures as a rule are readily found. The newly formed epithelial cells are desquamated often in large numbers and seem as phagocytic as endothelial cells, which they resemble in every way. The best proof that epithelial cells are phagocytic is the fact that they often can be found containing cell inclusions while they still are attached to the walls of the alveoli.

It is to be noticed that the epithelial cells which have been demonstrated to proliferate and to become phagocytic under the action of bacterial toxines are all cells of a low, undifferentiated type; they are flat cells which resemble endothelial cells and probably perform much the same functions.

There are two situations in which I have found phagocytic cells where their origin is less easily determined.

In one kidney of a very young child (B. C. H., No. 98.233), who died of diphtheria complicated with focal pneumonia and suppuration of both middle ears, there were found several small grayish areas, the largest $4 \mathrm{~mm}$. in diameter, running from the papillæ of the pyramids into the cortex. The areas were rather soft but not fluid; in places small hæmorrhages had taken place into them. Unfortunately no cultures were made from these small areas which microscopically showed an acute pyelonephritis. The tubules in the pyramids were dilated and filled chiefly with phagocytic cells; a few polynuclear leukocytes were present, but most of them were enclosed within the phagocytes. In the connective tissue between the tubules there were many phagocytic cells and some polynuclear leukocytes, lymphoid and plasma cells. In the cortex the areas consisted almost entirely of phagocytic cells lying in the tubules and in the intertubular tissue. In some places all evidence of tubules had disappeared and there existed large areas of phagocytic cells with here and there a few polynuclear leukocytes, and lymphoid and plasma cells betweeen them. In the pyramids many of the phagocytic cells contained large numbers of bacilli which in their morphology and staining reactions resembled in every respect the colon bacillus. The organisms also occurred to some extent outside of the cells; in the cortex they were much fewer in number and occurred mostly in clumps. 
So far as could be determined from a study of the sections the phagocytic cells arose entirely in the intertubular tissue. Several of them were found immigrating into the tubules, having passed but part way through the basement membrane. They were also often present in numbers between the renal cells and the basement membrane, apparently having pushed the cells from the wall. In other instances they had distended the lumina of the tubules and flattened the epithelial cells.

The second case is more difficult. In the epididymis of an actively functioning testicle removed from a man 72 years old in consequence of hypertrophied prostate, there were found great numbers of perfectly formed spermatozoa and also numerous huge phagocytic cells incorporating and digesting the spermatozoa by the hundreds. These phagocytic cells cannot be polynuclear leukocytes because the latter are never phagocytic for other cells. They can be only epithelial or endothelial cells; the latter view seems the more probable because it is difficult to conceive of the rather highly differentiated epithelial cells lining the epididymis being transformed into phagocytic cells.

The question whether bacterial toxines can cause connective tissue cells to proliferate and to become phagocytic is not easy to demonstrate. The best evidence is furnished, perhaps, by tuberculosis, but it is not conclusive. It is easy to show that a good deal of connectivetissue reticulum is formed between the epithelioid cells in the periphery of a miliary tubercle and also outside of the tubercle, but that does not prove that the epithelioid cells have produced that reticulum and therefore necessarily are connective-tissue cells. Wherever lymphoid and plasma cells are collected in numbers, a reticulum always forms between them. In the more chronic forms of capsular glomerulonephritis, a connective-tissue reticulum extends in from the capsule and out from the glomerular tuft, and spreads gradually between the epithelial cells in places forming a sort of basement membrane on which they tend to arrange themselves. In this way the capsular space is often divided into a number of small gland-like cavities. In neither of these two cases is it possible for the connective-tissue reticulum to have been produced by the cells which lie in its meshes. 
The reticulum seems to grow in between the cells because there is a physiological need of it.

It is not improbable that a good many of the phagocytic cells in the typhoid lesions of the intestine, especially in the muscular coat, are derived from connective-tissue cells; but it is not easy to demonstrate it, partly because endothelial cells occur practically everywhere, partly because the connective-tissue cell, away from the intercellular substance it produces, possesses no very definite characteristics.

In connection with the subject of this paper the following case (B. C. H., No. 98.252) is of interest. A woman 48 years old, with alcoholic history, died of hypertrophic cirrhosis after symptoms lasting about seven months. The inner surface of the bladder except at the neck was found everywhere studded with slightly elevated, flattened, grayish to yellowish-gray nodules of a rather translucent appearance, varying in size $\mathrm{frcm} 1 \mathrm{~mm}$. to $2 \mathrm{~cm}$. in diameter. Only occasionally were the edges of the nodules overhanging. They projected from 1 to $4 \mathrm{~mm}$. above the surface, and seemed to lie wholly within the mucous membrane as they were freely movable over the underlying muscle tissue. Two or three of the nodules showed slight hæmorrhages. So far as could be made out the nodules were not subdivided into papillæ, $i$. e. they did not present a cauliflower appearance. The mucous membrane over the nodules was smooth and glistening.

The condition was diagnosticated at first as multiple papillomata of the bladder.

Microscopic examination of the nodules after fixation of the tissue in Zenker's fluid, three hours after death, showed that they were due to collections of large phagocytic cells in the lymph spaces of the upper part of the submucosa. The cells were always most abundant just beneath the epithelium; in the smaller nodules they were confined to this situation, but in the larger nodules they extended more or less deeply into the submucosa, but never so far as to reach the underlying muscle tissue. The capillaries and larger blood-vessels in the nodules were more or less congested, and the tissue around them showed marked infiltration with lymphoid and plasma cells. 
There were also numerous lymphoid and plasma cells beneath the mucous membrane and around the vessels between the nodules. The epithelium of the mucous membrane was usually well preserved. Over the nodules it was much thinned, and in places was reduced to a single layer of cells or was even missing.

The cells in the nodules varied a good deal in size, but usually were very large, and round, polygonal, or irregular in shape; the protoplasm as a rule was homogeneous and stained rather deeply with eosin. The nuclei usually single but sometimes two in number, were vesicular in character, round, oval, or irregular in shape, and usually eccentrically situated.

The inclusions in these large cells were of two sorts, bacteria and cells. The bacteria were short rods, in general occurring in small closely aggregated clumps which often lay within vacuoles. The number of organisms in a clump varied from half a dozen to dozens. Some cells contained as many as 7 or 8 clumps of organisms. Other cells contained from a few to hundreds of these organisms scattered diffusely in the protoplasm. The bacteria were found almost entirely within the phagocytic cells; some of them stained sharply, others faintly; many were evidently dead and stained faintly or deeply with eosin. In the cells where the organisms seemed to be growing most rapidly they were usually shorter and plumper than in the cells which contained fewer of them. Occasionally short chains of 2 to 4 members were found. The Gram staining method gave negative results. It is impossible of course to say what the organism is. Morphologically and tinctorially it belongs to the colon-typhoid group.

In places, especially within the larger nodules, nearly every phagocytic cell contained organisms. In other places they were not found in more than one cell in twenty. They were always more numerous near the surface of the nodules than in the deeper portions.

The cells included in the phagocytes were chiefly polynuclear leukocytes and lymphoid cells. Occasionally red blood-globules were incorporated. Most of the inclusions were so far digested that their nature could not be made out. The number of inclusions in a cell varied greatly, but rarely exceeded four or five, although occasionally 
there were over a dozen. As a rule the inclusions lay within vacuoles. Within the protoplasm of many of the phagocytic cells were small hyaline bodies of varying size, stained pink and lying in vacuoles. Evidently they were the remains of incorporated cells. Many of them resembled closely the inclusions in the cells of carcinoma.

The nature of these phagocytic cells is difficult to determine absolutely. They resemble in all respects the phagocytic cells found in the lesions of typhoid fever. In all probability they are endothelial cells; but it is difficult to exclude entirely an origin from connectivetissue cells. Where the phagocytic cells were numerous, very little evidence of connective tissue could be found. At the bases of the nodules single phagocytic cells were present in the submucosa lying apparently in spaces between the strands of fibrous tissue. The inclusions in this situation consisted chiefly of hyaline, often concentrically layered, refractive, spherical bodies. The cells often showed forms suggestive of amœeboid motion. They seemed to lie in lymph spaces. Distinct lymphatic vessels were very few in number; they contained phagocytic, lymphoid, and plasma cells. In the mucous membrane covering the nodules, phagocytic cells were often found, sometimes in considerable numbers, between the epithelial cells. They probably had migrated to that situation from the underlying tissue. Occasionally in some of the nodules there were small collections of leukocytes, attracted, so far as could be made out, by degeneration of phagocytic cells.

It seems reasonable to conclude that these tumor-like nodules composed of phagocytic cells lying in the submucous tissue of the bladder are due to the action of the organisms found in such large numbers within many of the cells. Certainly the nodules have none of the characteristics of a true tumor formation.

So far I have considered the action of toxines on only three forms of cells. Their action on three other forms I will mention briefly.

The great increase in the number of the polynuclear leukocytes in the blood in infections with certain bacteria, especially the pyogenic organisms, is well known. The leukocytosis produced is of diagnostic 
importance; so also is the increase in the number of eosinophiles in trichinosis. ${ }^{3}$

Of greater significance in this connection is the increase of lymphoid and plasma cells which takes place in certain cases of scarlet fever and diphtheria. This has been considered at length by Councilman in his paper on Acute Interstitial Nephritis. ${ }^{4}$ The cells collect in the veins of the pyramids in the kidney; they emigrate from the vessels into the intertubular tissue and continue to proliferate there; by their numbers they interfere with the function of the kidney and may cause destruction of the tubules. The process in some respects closely resembles a malignant growth, such as leukæmia or lymphosarcoma.

The tendency of the cells which proliferate under the action of toxines is to degenerate and disappear as soon as the agent to which they owe their existence is destroyed or is neutralized. The best example is afforded, perhaps, by the resolution which takes place in the hyperplastic patches of Peyer and in the mesenteric lymph nodes in typhoid fever. The affected tissues quickly recover their normal appearance. In certain situations the results of the proliferation are more permanent and more disastrous. For example, the renal glomeruli may be destroyed partially or entirely by occlusion of the capillaries, or by the formation of fibrous tissue in the capsular spaces, and be converted into small contracted masses of hyaline connective tissue.

The processes of proliferation and phagocytosis which I have been describing as due directly to the action of toxines are regarded generally as reparative in nature. Similar processes certainly occur in repair. Is it possible to differentiate them?

In repair, cells proliferate for definite purposes-epithelium to cover denuded surfaces; connective tissue and lymph endothelial cells to replace losses of tissue and to remove foreign bodies, such as necrotic cells, fibrin, fat, myelin, etc.; blood endothelium to form new bloodvessels for the nourishment of the new tissue.

The cells, however, which proliferate under the direct action of

${ }^{3}$ Brown, Trichinosis, with expecial reference to the increase of the eosinophilic cells in the blood and muscle. Journal of Experimental Medicine, 1898, iii, p. 315.

4 Jounal of Fxperimental Medicine, 1898, iii, p. 393. 
toxines, multiply greatly in excess of need, and show a lack of definite purpose; they may exert some more or less beneficent action, such as possibly the production of antitoxines, but they can no more be regarded as reparative than the exudation called out by the toxines; both may work infinite harm. As we have seen in the cases cited, phagocytic cells may block up lymphatics and undergo necrosis; they may be carried to the liver as emboli, blocking up the capillaries and giving rise to focal necroses of liver cells; they may enclose and destroy the lymphoid cells in lymph nodules and then undergo necrosis themselves; they may occlude the veins in the spleen, giving rise to infarctions; they may occlude the capillaries of the glomeruli by growth within the vessels or by pressure from without; they may give rise to tumor-like formations. Lymphoid and plasma cells may multiply in the circulation, and may invade and multiply in the kidney interfering with its functions and causing its destruction. The phagocytic cells are phagocytic beyond all bounds of necessity and destroy great numbers of active, useful cells. These are all abnormal and to a certain degree malignant properties. 